\title{
Infrared Polarizing Reflectarray Metasurfaces
}

\author{
Mehdi Veysi, Caner Guclu, Ozdal Boyraz, and Filippo Capolino* \\ Department of Electrical Engineering and Computer Science, \\ University of California Irvine, CA, 92697, USA \\ *f.capolino@uci.edu
}

\begin{abstract}
An infrared-frequency polarizing reflectarray is designed with a phase-amplitude synthesis method at $4 \mu \mathrm{m}$ wavelength. The proposed phase-amplitude synthesis method is based on the optimization of the axial ratio of the steered beam through the reflection phase and amplitude of reflectarray elements. The proposed reflectarray reradiates an incoming slant linearly polarized wave into a circularly polarized reflected wave with an axial ratio less than $0.8 \mathrm{~dB}$ in the main beam direction.
\end{abstract}

\section{INTRODUCTION}

Techniques of beam shaping through phase discontinuities achieved by collections of resonant antennas have been used at centimeter and millimeter waves for a few decades [1-3]. In recent years, introducing the generalized Snell's laws [4] at infrared/optical frequencies once again turned researchers' attention to the flat plasmonic metasurfaces and, more specifically, to the infrared reflectarrays. Although, several infrared reflectarrays have recently been demonstrated [5-9], design of infrared reflectarrays is still a new and challenging subject due to material losses and the limitation introduced by the fabrication bottlenecks. At microwave frequencies, losses in substrates and conductors are negligible and return loss is very small, therefore the reflection amplitude on each reflectarray element are almost the same. In other words, the amplitude of the reflected field is mainly determined by the feed pattern [1-2]. As a result, since the element phase is the only variable to be optimized, the synthesis of the reflectarray at microwave frequencies has been defined as a phase-only synthesis. In contrast, the substrate and metal losses [6] are important considerations in birefringent reflectarray designs at infrared frequencies. From magnitude curve investigations, it can be concluded that amplitude of the reflection coefficient changes a lot depending on the design parameters values and inherent losses. Thus, the synthesis of reflectarrays at infrared frequencies cannot be just a phase only synthesis method since both the amplitude and phase of the reflected wave on the reflectarray surface should be optimized simultaneously.

In this paper, we apply an efficient phase-amplitude synthesis technique for circularly polarized reflectarrays at infrared frequencies. The proposed reflectarray is composed of anisotropic Y-shaped nanoantennas placed on top of a grounded stand-off material. The $\mathrm{Y}$-shaped nanoantennas are designed to locally manipulate the polarization and phase front of the incoming wave. The most challenging part of the task is to consider the loss and plasmonic features of the metals [6]. Especially, for polarizing reflectarray design, we should definitely take the magnitude of the reflection coefficient into account. Although low-cost conductors such as aluminum have a higher loss than noble metals in general, here the aluminum nanoantennas is chosen for their importance in fabrication technology and also with the aim of studying a worst-case scenario in which the loss plays an important role as a function of the $\mathrm{Y}$-shaped nanoantenna dimensions.

\section{REFLECTARRAY DESIGN}

The polarizing reflectarray design is composed of two main steps (i) polarization conversion and (ii) synthesizing the reflected wave phase-front. Here we start with the theory of polarization control as follows. Let us take a MS on $x-y$ plane, and assume that it is illuminated by a normally incident plane wave propagating along the $-z$ direction with electric field phasor $\mathbf{E}^{i}=\left(E_{x}^{i} \hat{\mathbf{x}}+E_{y}^{i} \hat{\mathbf{y}}\right) e^{j k_{0} z}$, where $k_{0}$ is the free-space wavenumber, $E_{x, y}^{i}$ are complex amplitudes of $x$ and $y$ components. In general, in a reflectarray regime, the reflected wave (the fundamental Floquet wave) takes the form $\mathbf{E}^{r}=\left(E_{x}^{i} \Gamma_{x} \hat{\mathbf{x}}+E_{y}^{i} \Gamma_{y} \hat{\mathbf{y}}\right) e^{-j k_{0} z}$, where $\Gamma_{x, \mathrm{y}}$ is the reflection coefficients pertaining to $x$ and $y$ components respectively. Note that the aim is to convert an incident wave with an arbitrary polarization (determined by $\left|E_{x}^{i} / E_{y}^{i}\right|$ and $\left.\angle\left(E_{x}^{i} / E_{y}^{i}\right)\right)$ to a reflected wave with the desired polarization (determined by $\left|\left(\Gamma_{x} E_{x}^{i}\right) /\left(\Gamma_{y} E_{y}^{i}\right)\right|$ and $\angle\left(\Gamma_{x} E_{x}^{i}\right)-\angle\left(\Gamma_{y} E_{y}^{i}\right)$ ), by tuning $\Gamma_{x, y}$ in both phase and amplitude.

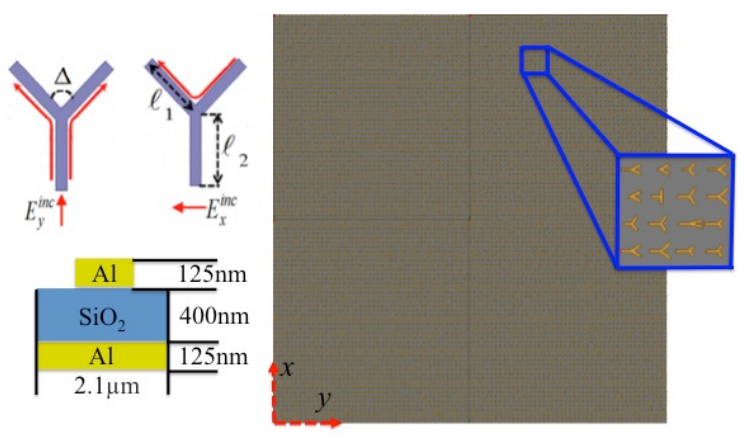

Fig. 1 Schematic of a Y-shaped nanoantenna with the current paths of symmetric and asymmetric modes (top left), the side view of each MS cell (bottom left). Schematic of the reflect array with size $215 \mu \mathrm{m}$ by $215 \mu \mathrm{m}(50 \lambda \times 50 \lambda)$ (right). 
For this purpose, we rely on arrayed nanoantennas to engineer $\Gamma_{x, \mathrm{y}}$ independently, and thus achieve the polarization conversion. To achieve linear to circular polarization conversion one should satisfy the condition $E_{x}^{i} \Gamma_{x}= \pm i E_{y}^{i} \Gamma_{y}$ locally at each element. When illuminated by a $45^{0}$ slant-polarized beam, one has $E_{x}^{i}=E_{y}^{i}$, whereas the elements are to be tuned to achieve $\Gamma_{x}= \pm j \Gamma_{y}$. To this aim, an array of anisotropic Y-shaped nanoantenna elements is used as in Fig.1 (a). Therefore the polarizing reflectarray design procedure proposed here relies on a simulation-based optimization of physical parameters in order to guarantee obtaining the desired polarization upon reflection. One can tune $\angle \Gamma_{x}$ and $\angle \Gamma_{y}$ independently by adjusting the $\mathrm{Y}$-shaped resonator's dimensions, $l_{1}, \Delta$, and $l_{2}$ reported in Fig. 1(a). The elements are tuned using a frequency domain finiteelement method solver (Ansys HFSS) (in a fully periodic arrangement, using local periodicity assumption validated in reflectarray studies [1]. The optimization is carried out to pick the most suitable $\mathrm{Y}$ antenna dimensions for each element in order to satisfy the condition $\Gamma_{x}= \pm j \Gamma_{y}$.

\section{RESULTS AND DiscUSSION}

A center-fed $215 \mu \mathrm{m}$ by $215 \mu \mathrm{m}$ sized polarizing reflectarray with focal length $f=172 \mu \mathrm{m}$ and main beam direction $\theta_{0}=30^{\circ}, \phi_{0}=0^{\circ}$, is designed at $\lambda=4 \mu \mathrm{m}$. A slant linearly polarized wave pattern represented by $\cos ^{q} \theta$ and originated from the focal point illuminates all the reflectarray's elements with a $10 \mathrm{~dB}$ taper illumination at the reflectarray's edges. An array of aluminum $\mathrm{Y}$-shaped nanoantennas is patterned on one side of a silica $\left(\mathrm{SiO}_{2}\right)$ substrate with thickness of $400 \mathrm{~nm}$, while a thin aluminum plane is patterned on the other side of the substrate. Fig. 1(b) shows the schematic of the designed polarizing reflectarray. Because of the large size of the polarizing reflectarray, it is not efficient to simulate the structure using a full wave solver. Thus, the Fast Fourier Transform (FFT) algorithm [1-2] is applied in this work to theoretically compute the circular polarization radiation pattern. This algorithm ignores the undesirable edge diffraction and specular reflection effects, which become less relative to the desirable circular polarized radiation, as the reflectarray size gets bigger. The simulated circularly polarized radiation pattern and axial ratio of the metasurface prototype are shown in Fig. 2. As can be seen, the simulated axial ratio level of the reflectarray is quite small (less than $0.8 \mathrm{~dB})$ around the main beam direction $\left(\mathrm{U}_{0}=0.5\right.$, and $\left.\mathrm{V}_{0}=0\right)$. Note that the anisotropic Y-shaped nanoantennas are also good candidates for designing dual beam linear orthogonally polarized reflectarrays due to their excellent engineered birefringent behaviors.

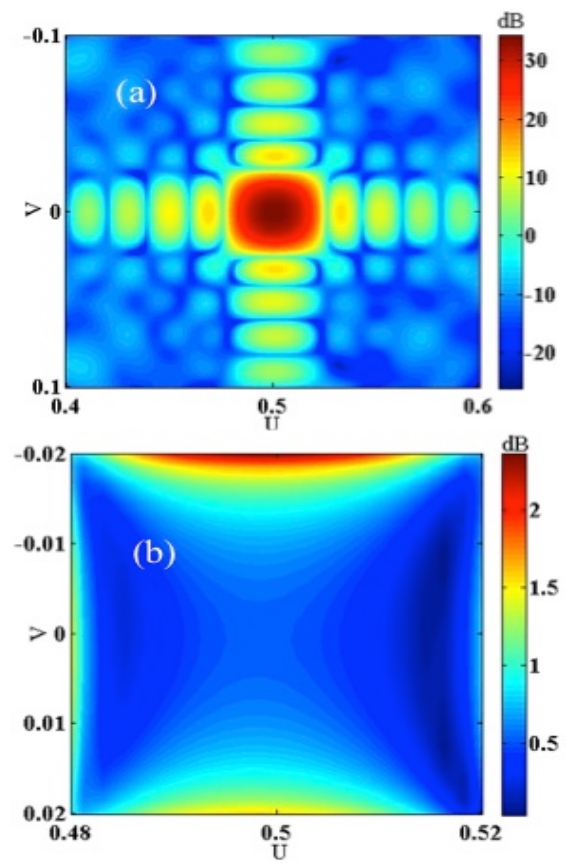

Fig. 2. Far-field LHCP wave's (a) directivity and (b) axial ratio of the designed infrared polarizing reflectarray in $\mathrm{U}-\mathrm{V}$ plane where $U=\sin \theta \cos \phi$ and $V=\sin \theta \sin \phi$.

Acknowledgement

The authors thank ANSYS, Inc., for providing HFSS.

\section{REFERENCES}

[1] J. Huang, and J. A. Encinar, "Reflectarray antennas" John Wiley \& Sons, Inc., 2008.

[2] J. A. Zornoza, and J. A. Encinar, "Efficient phase-only synthesis of contoured-beam patterns for very large reflectarrays" Int. J. RF and Microwave Computer-Aided Eng. Vol. 14, pp. 415-423, 2004.

[3] H..Hasani, J. Perruisseau-Carrier, C. Peixeiro, J.R. Mosig, "Dual-band dual-polarized reconfigurable unit-cell for reflectarray antenna in $\mathrm{Ku}$ Band" 7th European Conference on Antennas Propag, (EuCAP), pp. 861-862, 2013.

[4] N. Yu, P. Genevet, M. A. Kats, F. Aieta, J. Tetienne, Gaburro, "Light propagation with phase discontinuities: generalized laws of reflection and refraction" Science, vol. 334, no. 6054, pp. 333-337, Sep. 2011.

[5] J. C. Ginn, B. A. Lail, and G. D. Boreman, "Phase characterization of reflectarray elements at infrared" IEEE Trans. Antennas Propag., vol. 55, No. 11, pp. 2989-2993, Nov. 2007.

[6] F. Yang, P. Nayeri, A. Elsherbeni, Y. Rahmat-Samii, "Reflectarray design at infrared frequencies: effects and models of material loss" IEEE Trans. Antennas Propag., vol. 60, No. 9, pp. 4202-4209, Sep. 2012.

[7] T. Niu, W. Withayachumnankul, B. S. Y. Ung, H. Menekse, M. Bhaskaran, S. Sriram, and C. Fumeaux, "Experimental demonstration of reflectarray antennas at terahertz frequencies" Opt. Express vol. 21, Issue. 3, pp. 2875-2889, 2013.

[8] E. Carrasco, M. Tamagnone, and J. Perruisseau-Carrier, "Tunable graphene reflective cells for $\mathrm{THz}$ reflectarrays and generalized law of reflection" Applied Phys. Lett. Vol. 102, Issue. 10, pp. 104103-104104, Mar. 2013.

[9] M. Farmahini-Farahani and H. Mosallaei, "Birefringent reflectarray metasurface for beam engineering in infrared" Opt. Lett. Vol. 38, Issue. 4, pp. 462-464, 2013. 